\title{
Chasseurs dans le monde kalam
}

\section{Denis Monnerie}

\section{OpenEdition}

Journals

Édition électronique

URL : https://journals.openedition.org/jso/5966

DOI : 10.4000/jso.5966

ISSN : 1760-7256

\section{Éditeur}

Société des océanistes

\section{Édition imprimée}

Date de publication : 15 décembre 2009

Pagination : 295-300

ISBN : 978-2-85430-026-0

ISSN : 0300-953x

\section{Référence électronique}

Denis Monnerie, «Chasseurs dans le monde kalam », Journal de la Société des Océanistes [En ligne], 129 | juillet-décembre 2009, mis en ligne le 15 décembre 2009, consulté le 09 mars 2022. URL : http:// journals.openedition.org/jso/5966; DOI : https://doi.org/10.4000/jso.5966

\section{(c) $\oplus \Theta \Theta$}

Journal de la société des océanistes est mis à disposition selon les termes de la Licence Creative Commons Attribution - Pas d'Utilisation Commerciale - Pas de Modification 4.0 International. 


\title{
MISCELLANÉES
}

\section{Chasseurs dans le monde kalam}

par

\author{
Denis MONNERIE*
}

À propos de MajneP Ian Saem et Ralph BuLMER, 2007. Animals the Ancestors Hunted. An account of the wild mammals of the Kalam area, Papua New Guinea, Adelaide, Crawford House Publishing Australia. 452 p., 18 illustrations de Christopher Healey, 15 photographies, 3 cartes, 3 glossaires, 3 index.

C'est un livre de chasseur et de savant que nous donnent à lire les regrettés Ian Saem Majnep et Ralph Bulmer, dont la collaboration a profondément marqué l'ethnologie et en particulier «l'ethnobiologie» de la Papouasie Nouvelle-Guinée (Pawley, 1991). Cet impact s'était fait tout particulièrement sentir avec un premier grand livre, Birds of my Kalam Country (1977), ouvrage à deux voix, où l'ethno(zoo)logue Bulmer et son interlocuteur néo-guinéen Majnep nous présentaient, chacun de son point de vue, l'avifaune du pays kalam. Leurs deux voix étaient enrichies du regard de Christopher Healey avec de magnifiques illustrations que l'on retrouve dans ce nouvel ouvrage (hélas ici encore reproduites en noir et blanc, vraisemblablement en raison du dénuement financier de l'édition anthropologique). Animals the Ancestors Hunted est ainsi le second volet d'une œuvre majeure - en attendant son parachèvement avec la publication du dernier livre de la trilogie : Kalam Plant Lore.
Animals the Ancestors Hunted diffère du livre précédent : alors que, pour chaque grande partie, la plupart des brèves introductions sont de la plume de Bulmer, l'essentiel de la présentation des animaux est de Majnep et constitue le gros du texte. Le travail de Bulmer consiste ici surtout en transcription, traduction et édition du texte, en collaboration avec Saem. Les difficultés de ces tâches (pp. xv-xviii, xxiv-xxvi) furent en partie à l'origine d'une première publication en langue kalam, destinée autant à un public académique qu'aux jeunes générations de cette population (Majnep, 1990). Voisins des Maring et des Melpa, dont les études sont des classiques de l'ethnologie de la Papouasie Nouvelle-Guinée, les Kalam vivent dans la Province de Madang à la limite des Hautes Terres occidentales. Animals the Ancestors Hunted est un livre précieux, passionnant et difficile. Précieux par l'abondance des savoirs qu'il recèle. Passionnant parce que Majnep décrit ces gibiers, leurs environnements, leur vie, les tactiques des chasseurs et chasseuses et celles de défense des animaux, leurs qualités alimentaires et leurs usages rituels ou quotidiens dans des évocations savantes, mais fluides, concrètes, précises, inattendues souvent aussi. Son style oral est magnifiquement rendu par la traduction anglaise. Livre difficile parce qu'il ne

\footnotetext{
* Professeur d'ethnologie, Université de Strasbourg, Département d'ethnologie, Laboratoire Cultures et sociétés en Europe (CNRS-Uds), monnerie@unistra.fr
} 
nous tranquillise pas en offrant une interprétation ethnologique construite de ces connaissances - mais nous incite à tenter de devenir, le temps d'une lecture, ethnologue des Kalam, un vrai casse-tête! Car c'est bien ici la voix de Majnep qui domine l'ensemble, le dialogue avec les conceptions scientifiques occidentales prenant surtout la forme d'introductions, de notes et d'appendices.

Fils d'une veuve - à qui les Kalam ont réservé, avec ses enfants, un sort peu enviable (pp. 9-17)-, Majnep a dû survivre dès l'âge de trois ou quatre ans de chasse et de cueillette, avec sa mère, à qui il dédie ce livre, et qui le porta quand ils dormaient « sous les arbres, dans les abris rocheux et sous les toits crevés, ruisselants, de maisons abandonnées, lugubres ». Avec ses frères aînés, des oncles et des tantes aussi, cette femme lui enseigna la chasse, ce qui fait de ce livre un document très complet qui intègre les pratiques cynégétiques des hommes et des femmes du pays kalam. Livre d'un chasseur et d'un savant donc, à qui Bulmer a demandé de choisir son sujet et son titre. Majnep décida de traiter en priorité, non pas des plantes, mais des kmn (kalam) ou kapuls (pidgin), catégorie qui inclut nombre de mammifères (et des marsupiaux), à l'exception notable des chauves-souris (classées avec les oiseaux, yakt), porcs, chiens et autres grands quadrupèdes importés. Outre les kmn, le livre présente de petits rongeurs classés par les Kalam comme as - avec les batraciens - et des kopyak, rats, se nourrissant d'ordures et de patates douces et qui, pour les Kalam, forment une catégorie marquée par des interdits de nourriture surprenants - les Maring, eux, mangent ces mêmes rats (pp. 249-251). Regroupés en douze parties, les vingt-deux chapitres du livre présentent un animal principal, souvent associé, par les Kalam, à d'autres qui partagent avec lui certains traits significatifs. La treizième et dernière partie de l'ouvrage présente des plantes sauvages avec leur utilisation dans le contexte de la chasse et un appendice de près de cinquante pages regroupe « les plantes significatives dans les savoirs kalam sur les animaux » (pp. 349-391).

Plutôt que de présenter l'ensemble de ces chapitres, je résumerai l'un d'eux, qui témoigne bien des spécificités du livre, que ce soit pour la qualité des informations ou par la façon de les présenter. Dans la cinquième partie de l'ouvrage introduite, non par Bulmer, mais par Robin Hide et Andrew Pawley -, le chapitre 11 est consacré au wgi, bandicoot à longue queue (illustré par Healey) et au bandicoot chasseur. Majnep décrit les variétés du premier de ces marsupiaux. Il passe alors à son comportement vis-à-vis des petits :

« un jeune mâle est expulsé du nid [...] avant une jeune femelle. La raison en est que la mère craint que, si son rejeton mâle reste [...] il puisse essayer de copuler avec elle, alors elle s'en débarrasse. » (p. 177)

Suit une description très précise des environnements favorisés par l'animal, de son nid qui comporte «une entrée principale» dissimulée par du feuillage mais aussi «un tunnel de secours » permettant une fuite vers les galeries souterraines de l'undercroft (voir infra). Ici, les femmes, qui chassent à proximité de la surface du sol, détiennent la plus grande expertise, car la plupart des hommes chassent dans les arbres, parfois à très grande hauteur. Ensuite viennent les nourritures de l'animal, ses traces, la façon de le piéger, de le mettre à mort, les tableaux de chasse (de cinq à quinze individus), ses différentes préparations culinaires - quotidiennes ou cérémonielles -, les précautions psychologiques, magiques ou de communication qui visent au succès de la chasse. Majnep évoque aussi ses souvenirs d'enfance liés à cette chasse et aux lieux préférés de sa mère. Nous apprenons ensuite qui la pratique ou non et comment elle est enseignée. Viennent alors les interdits de consommation : « un spécialiste du rituel [...] ne consomme jamais cet animal, ne l'a jamais fait et ne le fera jamais ", il ne mangera rien qui ait été cuit sur les mêmes pierres, pour lui, bien d'autres tabous encore s'appliquent à la viande de cet animal et certains s'étendent aussi aux hommes dès l'adolescence (pp. 182-183). Pour Majnep, le $w g i$ est aussi support de souvenirs d'enfance. De façon générale, les Kalam pensent que « les jeunes enfants nourris avec cette nourriture deviennent grands et forts » (p. 183). Cette section s'achève avec des considérations sur les saisons privilégiées de la chasse, les compétences comparées des chasseuses selon leur milieu écologique de résidence et les pratiques de partage. Saemévoque enfin les plantes utilisées pour farcir l'animal - avec, à ce sujet, la description d'une autre prescription, et non des moindres : aux enfants la viande, aux adultes la farce. Les $w g i$ sont, on le voit, un gibier important autour duquel s'articulent enfance et maturité, dans plusieurs domaines d'expérience significatifs. À la fin du chapitre, le bandicoot chasseur, considéré par les Kalam comme proche du wgi, est l'objet d'un traitement plus rapide, surtout fait de comparaisons : de taille, de forme, d'odeur, d'habitat, d'habitudes et d'interdits de consommation.

Revenons à l'undercroft (en kalam, abn, les cavités). Ce néologisme créé par Bulmer ne pos- 
sède pas non plus je crois d'équivalent français. Constitué par les racines supérieures des arbres de la forêt primaire et leur compost, l'undercroft est bordé pour les humains par une surface sur laquelle on marche comme sur un sol homogène mais qui, en fait, cache, à peu de profondeur, un important réseau de galeries souterraines où vivent, se déplacent ou se réfugient nombre de petits animaux. Un monde souterrain dont, nous dit Bulmer, les Occidentaux, y compris les écologues, ne soupçonnent pas la complexité (p. 43). On a ici, dans un contexte cynégétique, un élément d'une conceptualisation « verticale » du monde, organisée autour de la surface ; celleci, avec l'undercroft, est le domaine de chasse privilégié par les femmes, alors que le domaine des hommes est, vers le haut, celui des grands arbres. Ce type de conceptualisation verticale du monde se retrouve au plan cultural, cérémoniel ou rituel dans certaines sociétés de Mélanésie (Coppet, 1976 ; Sahlins, 1995 : 160 ; Monnerie, 1995, 1996 ; Juillerat, 2001 : 210-211). Cependant, pour les Kalam, elle n'est pas explicitée en tant que telle par Majnep, mais apparaît souvent en filigrane dans le contexte de la chasse.

À propos des oiseaux kalam, Majnep, dans les premières années de sa collaboration avec Bulmer, déclarait :

« Je crois que vous avez un gros problème pour décider comment ordonner tout ceci. »

Ce genre d'interrogation a mené à la rédaction de l'article le plus connu de celui-ci qui rend compte du fait que, pour les Kalam, le casoar n'est pas un oiseau (1967), suivi d'une série d'articles confrontant savoirs kalam et connaissances scientifiques occidentales (Bulmer, 1968, 1974, 1979 ; Bulmer and Menzies, 1972 ; Bulmer and Tyler, 1968 ; Bulmer, Menzies and Parker, 1975). À mon sens, ce « gros problème » perdure et il s'étend au-delà de la seule question des classifications. Pour comprendre le monde kalam, une large part des travaux de Bulmer et Majnep (avec leurs autres collaborateurs) propose des matériaux et des réflexions d'une qualité et d'une précision rares, généralement en les juxtaposant avec les représentations scientifiques naturalistes. Cette démarche comparatiste permet aux spécialistes d'avancer dans la connaissance des questions d' " ethnobiologie » ou, plus précisément, des relations des Kalam à leur univers, tout en faisant bien apparaître la complexité de cette entreprise. Car ces connaissances serrées s'intègrent difficilement aux propositions scientifiques actuelles, qu'elles soient naturalistes ou ethnologiques. Dans cette veine, Animals the Ancestors Hunted introduit des matériaux ethnographiques qui représentent un défi pour les anthropologues ; je voudrais consacrer la suite de cet à propos à des remarques qui soulignent la qualité de ce défi.

Animals the Ancestors Hunted suggère de nombreuses (re)lectures des relations des Kalam avec leur univers et, en particulier, avec le gibier. L'ouvrage présente une ethnographie qui permet d'aller au-delà des éléments d'analyse qui y sont proposés, en particulier par Bulmer. Dans une note de travail, ce dernier avait remarqué " qu'il serait difficile d'ordonner ces animaux » d'une façon plus différente de celle de la taxinomie zoologiste actuelle (p. xxix). En effet, comme pour les oiseaux, les considérations spatiales et écosystémiques - dans leur interprétation kalam - jouent un rôle considérable dans la façon dont Majnep présente les kmn. Il me semble qu'on peut dégager un premier principe général, au fond assez simple: les animaux sont connus principalement en relation avec les espaces où ils vivent et qu'ils partagent peu ou prou avec les Kalam. Cette forme de découpage emic des espaces, paysages et écosystèmes, de façon générale, semble revêtir une importance toute particulière pour nombre de peuples des Hautes Terres de Papouasie Nouvelle-Guinée (Sillitoe, 1998; Brunois, 2008). Comme le montrent les connaissances concernant les galeries souterraines, les ethnologues et les naturalistes ont encore à apprendre dans ces domaines. Mais la question se complique quand on cherche à aller au-delà de cette caractérisation spatiale et écologique large de l'organisation du monde kalam. Marshall Sahlins a consacré plusieurs pages très synthétiques à ces questions (Sahlins, $1995:$ 157-163) dans un livre qui conteste avec passion et conviction l'idée de Gananath Obeyesekere (1992) selon laquelle tous les peuples partageraient une rationalité pratique fondée sur l'expérience sensorielle. Alors que les propositions « réalistes" d'Obeyesekere "prétendent être déterminées par les choses en elles-mêmes et pour ellesmêmes ", Sahlins, lui, souligne que chaque épistémologie locale est " complètement imbriquée et médiatisée par l'ordre culturel local » (1995: 158). Les études sur les Kalam fournissent l'essentiel de son argumentation. Pour lui, une des clés du système met en œuvre des « distinctions morales humaines [human-moral distinctions] corrélées avec l'habitat et associées spécifiquement au monde des esprits » (Sahlins, 1995 : 161). Toutefois, ce schème général laisse de côté l'importance de l'expérience olfactive qu'il souligne pourtant au début de sa réflexion (Sahlins, 1995 : 158) et à laquelle, me semble-t-il, peu de distinctions « morales » sont attachées - l'appé- 
tence et le dégoût entrent-ils pour Sahlins dans cette catégorie ? Enfin, d'autres grands principes contribuent à organiser la présentation de Majnep, en particulier la prise en considération de la taille des animaux et les usages et interdits afférant aux nourritures - celles des humains mais aussi celles du gibier (pp. xxix-xxx). Pour reprendre l'exemple des wgi, une partie seulement de ces interdits relève d'analyses structurales, faisant intervenir des critères de genre, d'âge, de rapport au rituel, des oppositions comme intérieur-extérieur, viande-végétal, etc. Un problème, comme souvent en anthropologie, réside dans l'imbrication étroite des dimensions de distinction et de continuité ainsi que dans les superpositions et imbrications de systèmes.

Une autre réflexion s'impose. Majnep a sur les animaux une perspective de chasseur, le caractère serré de ses connaissances minimise vraisemblablement la place du hasard dans la démarche cynégétique. Cependant, dans l'approche qu'il nous propose du gibier, il n'y a guère de " perspectivisme » au sens d'Eduardo Viveiros de Castro (1998). Sur ce point, les Kalam se distinguent nettement, me semble-t-il, du monde amazonien. Faut-il y voir une différence "d'ontologie» entre ces peuples dans leurs modes d'identification des humains et des non-humains comme le propose un autre grand spécialiste de l'Amazonie, Philippe Descola (2004, 2005) ? Les thèses de Viveiros de Castro ont joué un rôle non négligeable dans l'élaboration de la réflexion théorique de Descola (sur ce point, voir l'intéressant article de Latour, 2009). Pour Descola :

« savoir chasser [...] c'est savoir adopter le point de vue de l'animal que l'on cherche. » (2005: 274)

Mais, à ce compte, le perspectivisme serait un trait universel implicite chez la plupart des chasseurs et pêcheurs du monde... Par ailleurs, nous n'avons aucune certitude sur le fait qu'un autre Kalam, aussi bon chasseur que Majnep, aurait classé ses descriptions exactement comme lui lui-même a changé plusieurs fois l'ordre de présentation de ses chapitres (p.xxix). Alors, n'est-ce pas la priorité donnée à la question de la catégorisation, de l'organisation, du découpage $\mathrm{du}$ monde (en particulier sous une forme statique, typologique, concernant les seuls animaux), qui pose problème et demande à être dépassée ? Jack Goody (1977) a bien souligné certains aspects généraux de ce biais. À vouloir en faire la pierre angulaire de nos interrogations, ne manquons-nous pas d'autres questionnements plus pertinents et scientifiquement plus productifs pour l'anthropologie?
C'est une des qualités des récents travaux de Philippe Descola dans son maître ouvrage, Pardelà nature et culture (2005) de nous proposer d'aller voir ailleurs :

« d'échapper à une interprétation trop classificatoire de phénomènes qui, à l'évidence, se prêtent mal à une telle lecture » (ibid. : 179)

et d'essayer de nouveaux angles d'approche, en particulier celui des divers modes d'identification possibles de l'homme avec son environnement - constitué de ce qu'il appelle des « existants ». Ce sont alors les «différentes manières dont nous disposons pour établir des continuités ou des discontinuités entre nous-mêmes et des éléments du monde » qui passent au centre de la réflexion (Descola, 2004 : 64-65). En opposition raisonnée aux thèses de Claude Lévi-Strauss, le livre de Descola s'ouvre sur une critique de la césure nature-culture qui mobilise aussi, parmi bien d'autres, les travaux de spécialistes de la Mélanésie, en particulier Roy Wagner, Edward Schieffelin, Daniel de Coppet et Marilyn Strathern. D'assez longue date, en effet, l'ethnographie de cette partie de l'Océanie a invité à une remise en cause de cette distinction et, dès 1947 , Maurice Leenhardt, comme le souligne bien Descola (2005 : 48-49), fut un des premiers à pointer la difficulté d'un tel clivage. Les ethnographies de Mélanésie invitent au moins à cette remise en cause et, mieux encore, à des tentatives de conceptualisation renouvelées. Leenhardt en fut un précurseur, avec l'idée de " cosmomorphologie » des sociétés de Mélanésie, poursuivie avec l'idée de « systèmes sociocosmiques » ou de « logique socio-cosmologique »; il s'agit de penser ensemble ce que les ethnologues désignent classiquement par "société, culture, nature et univers ", avec leurs interactions qui, en Mélanésie sont bien souvent d'échanges et de circulations (Barraud et al., 1984; de Coppet, 1976, 1981, 1990 ; Iteanu 1983 ; Monnerie, 1996 ; Juillerat, $2001:$ 159-178).

C'est une autre voie qu'emprunte Descola. La plus grande partie de son livre est consacrée à l'élaboration d'une vision universalisante des modes d'identification des humains et des autres existants, avec une typologie à quatre termes " animisme, naturalisme, totémisme, analogisme » qui structure la théorie de ces modes d'identification. Celle-ci se construit à partir d'une vaste réflexion sur les relations entretenues par "l'intériorité » des personnes et la " physicalité » du monde. Ici encore, Animals the Ancestors Hunted ne me semble pas se prêter facilement à une interprétation dans les termes de cette typologie. Avant tout, dans ce livre, Majnep 
ne nous donne guère d'indications permettant de définir une « intériorité » kalam. Plus largement, il nous manque souvent en Mélanésie de pouvoir bien cerner, à partir de l'ethnographie, ce que Descola nomme l'intériorité des personnes, celles-ci pouvant dans certains cas impliquer plusieurs composantes ou relations (Leenhardt, 1971 [1947] ; Coppet, 1981 ; M. Strathern, 1988 ; Monnerie, 1996) dont certaines établissent de façon différente les continuités et discontinuités avec des formes de "physicalité » - et surtout avec ces existants majeurs que sont les ancêtres. Que l'intériorité fasse problème pour Descola en Mélanésie est montré par sa brève discussion des thèses de Marilyn Strathern qui a proposé que l'on qualifie la personne, dans cette région du monde, non comme une individualité mais comme une "dividualité », définie en premier lieu par sa position et ses relations dans un réseau. Sans contester l'existence en Mélanésie d'une théorie de la personne " dividuelle », Descola affirme, avec Maurice Leenhardt autrefois et Edward LiPuma plus récemment, que celle-ci coexiste avec - et, dans certaines situations, est supplantée par - une conception plus égocentrée du sujet dont rien ne permet d'affirmer qu'elle serait le produit exclusif de la colonisation européenne (Descola, 2005: 170-171, voir aussi p. 168). Dans la définition de l'intériorité, il faudrait donc distinguer des plans; pour sa démonstration, Descola nous oriente vers un plan universel. Il définit cette intériorité commune par la combinaison de l'expression linguistique du « je » et du « tu » avec une expérience qui est « cette croyance universelle qu'il existe des caractéristiques internes à l'être ou prenant en lui sa source » (ibid. : 169). Dans cette quête d'une meilleure compréhension des relations des humains aux autres existants, si Animals the Ancestors Hunted doit nous servir de guide, c'est peut-être pour nous conduire vers la reconnaissance d'une diversité des positions des sociétés de Mélanésie par rapport aux schèmes de Descola - ce que ce dernier n'exclut, me semble-t-il, pas. En définitive, il faudra attendre la publication du troisième volume de la trilogie de Majnep et Bulmer, entièrement consacré aux plantes, pour que les collaborateurs de Majnep et Bulmer ou un non-spécialiste du terrain kalam, puissent envisager de proposer de façon circonstanciée des modèles ethnologiques des conceptions kalam de leur environnement. Celles-ci serontelles très différentes des propositions de Bulmer et ses collaborateurs, dont Sahlins a tenté de faire la synthèse ? Ou pourront-elles s'écarter des sentiers battus des études de classifications, pour suivre la voie ouverte par Descola? D'autres cohérences, d'autres voies d'accès seront-elles mises en évidence?

Au même titre que Birds from my Kalam Country, cet ouvrage est, je crois, indispensable à toute bibliothèque sérieuse, ethnologique ou naturaliste. Il est un outil de travail quasi irréprochable - les nombreux glossaires et index ne prêtent guère à critique, sauf peut-être pour l'absence de données précises et systématiques sur la taille des animaux mentionnés. Aussi, je n'en doute pas et je viens d'essayer de suggérer pourquoi ce texte pourra dérouter. L'incontestable plaisir de lecture ethnographique qu'il procure sera-t-il goûté par tous? Il met en évidence un certain " gai non savoir », proche de celui que procure cette " encyclopédie chinoise » de Borgès si souvent citée, en particulier, par Michel Foucault. Pour l'esprit non averti, peu réceptif ou fermement ancré dans la tradition française d'ironie - à la Georges Perec -, Animals the Ancestors Hunted ne sera peut-être qu'une sorte d'inventaire à la Prévert : bizarre, déconcertant, plaisant, grinçant peut-être, mais ne tirant guère à conséquence. Pour d'autres, il ménagera une béance, un souffle, bienvenus dans les certitudes sur le monde. Les passionnés qui s'essaient à la compréhension d'autres univers où sont tissés nature, culture, société et personne y trouveront un trésor de connaissances et d'aperçus sur le monde kalam, une fenêtre sur de nouvelles découvertes, de nouvelles perplexités, de nouvelles pistes.

\section{BIBLIOGRAPHIE}

Barraud Cécile, Daniel de Coppet, André Iteanu et Raymond JAmous, 1984. Des relations et des morts. Quatre sociétés vues sous l'angle des échanges, in Jean-Claude Galey (éd.), Différences, valeurs, hiérarchies, Paris, éditions de l'EHESs, pp. 421-520.

BRUNOIS Florence, 2008. Le jardin du casoar, la forêt des Kasua. Savoir-être et savoir-faire écologiques, Paris, CNRS Éditions/Éditions de la maison des sciences de l'homme.

BULMER Ralph, 1967. Why is the cassowary not a bird? A problem of zoological taxonomy among the Karam of the New Guinea Highlands, Man 2 (1), pp. 5-25.

_, 1968. Worms that Croak and Other Mysteries of Karam Natural History, Mankind 6, pp. 621-639.

_, 1974. Folk Biology of the New Guinea Highlands, Social Sciences Information 13, pp. 9-28.

_, 1979. Mystical and Mundane in Kalam Classifications of Birds, in Roy Ellen and David Reason (eds), Classifications in their social context, London, Academic Press, pp. 57-79. 
Bulmer Ralph and James MenzIes, 1972-1973. Karam Classification of Marsupials and Rodents, Journal of the Polynesian Society 81, 4, pp. 472-499 et 82, 1, pp. 86-107.

Bulmer Ralph, James Menzies et F. PArker, I 975. Kalam Classification of Reptiles and Fishes, Journal of the Polynesian Society 84, 3, pp. 267-308.

Bulmer Ralph and M. J. TYLER, 1968. Karam Classification of Frogs, Journal of the Polynesian Society 77, 4, pp. 333-385.

Coppet Daniel (de), 1976. Jardins de vie, jardins de mort en Mélanésie, Traverses 5-6, pp. 166-177.

_, 1981. The Life-Giving Death, in Sally C. Humphreys and Henry King (eds), Mortality and Immortality: the Anthropology and Archaeology of Death, London, New York, Academic Press, pp. 175-204.

-, 1990. The society as an ultimate value and the socio-cosmic configuration, Ethnos 3-4, pp. 140150.

Descola Philippe, 2004. Le monde, par-delà la nature et la culture, La Recherche 374, pp. 63-67.

—, 2005. Par-delà nature et culture, Paris, Gallimard.

GooDy Jack, 1977. The Domestication of the Savage Mind, Cambridge, Cambridge University Press (traduction française, 1979, La raison graphique, Paris, éditions de Minuit).

ITEANU André, 1983. La ronde des échanges. De la circulation aux valeurs chez les Orokaiva, Paris, Cambridge University Press - Éditions de la maison des sciences de l'homme.

Juillerat Bernard, 2001. Penser l'imaginaire, Lausanne, Payot.

LAtour Bruno, 2009. Perspectivism: "type" or "bomb"?, Anthropology today 25, 2, pp. 1-2.

LeEnHARdT Maurice, 1971 ( $1^{\mathrm{e}}$ éd. 1947). Do Kamo. La personne et le mythe dans le monde mélanésien, Paris, Gallimard.
MajneP Ian Saem, 1990. Kalam Hunting Traditions (I-VI), Auckland, University of Auckland, Department of Anthropology, Working Papers in Anthropology, Archaeology, Linguistics, Maori Studies 85-90.

Majnep Ian Saem and Ralph Bulmer, 1977. Birds of my kalam Country, Auckland, Auckland University Press.

MonNerIE Denis, 1995. On "Grand-Mothers", "Grand-Fathers" and "Ancestors": Conceptualizing the Universe in Mono-Alu (Solomon Islands), in Daniel de Coppet and André Iteanu (eds), Society and Cosmos in Oceania, Oxford, Berg, pp. 105-133.

_, 1996. Nitu, les vivants, les morts et le cosmos selon la société de Mono-Alu (Îles Salomon), Leyde, Center for Non Western Studies.

OBEYESEKERE Gananath, 1992. The Apotheosis of Captain Cook: European mythmaking in the Pacific, Princeton, Princeton University Press.

PAwley Andrew (ed.), 1991. Man and a Half: Essays in Pacific Anthropology and Ethnobiology in Honour of Ralph Bulmer, Auckland, The Polynesian Society.

SAHLIns Marshall, 1995. How "Natives" Think: About Captain Cook For Example, Chicago, The University of Chicago Press.

Sillitoe Paul, 1998. An Ethnobotanical Account of the Vegetation Communities of the Wola Region, Southern Highlands Province, Papua New Guinea, Journal of Ethnobiology 18 (1), pp. 103-128.

STRATHERN Marilyn, 1988. The Gender of the Gift: Problems with women and problems with society in Melanesia, Berkeley, University of California Press.

Viveiros de Castro Eduardo, 1998. Cosmological deixis and Amerindian perspectivism: A view from Amazonia, JRAI (ns) 4, pp. 469-488. 